PAPER

\title{
Risk-benefit analysis of the treatment of unruptured intracranial aneurysms
}

\author{
R R Vindlacheruvu, A D Mendelow, P Mitchell
}

J Neurol Neurosurg Psychiatry 2005;76:234-239. doi: 10.1136/jnnp.2003.031930

See end of article for authors' affiliations

....................

Correspondence to: P Mitchell, patrick. mitchell@ncl.ac.uk

Received 5November 2003 In revised form 28 April 2004 Accepted 25 May 2004

\begin{abstract}
Objectives: To determine under what circumstances repair of unruptured intracranial aneurysms may be beneficial.

Methods: A life expectancy analysis of patients with unruptured aneurysms with and without repair based on prospective data from the International Study of Unruptured Intracranial Aneurysms (ISUIA).

Results: Life years are lost at all ages by repairing anterior circulation aneurysms under $7 \mathrm{~mm}$ in diameter in patients with no history of a subarachnoid haemorrhage from another aneurysm (incidental). For all other aneurysms the number of life years saved by repair is dependent on the patient's age at the time when repair is undertaken. Between 2 and 40 years are saved by repairing aneurysms in patients aged 20 years. These benefits fall to 0 when remaining life expectancy falls below 15-35 years, corresponding to the age range of $45-70$ years.

Conclusions: Repair of unruptured aneurysms benefits patients harbouring them by improving life expectancy except in certain circumstances. The exceptions are patients with remaining life expectancy less than 15-35 years or aged 45-70 (depending on aneurysm size and location) and patients with aneurysms of the anterior circulation under $7 \mathrm{~mm}$ in diameter with no history of a previous subarachnoid haemorrhage. These results are based on the findings of the ISUIA and are dependent on their accuracy.
\end{abstract}

$\mathrm{T}$ he high mortality and morbidity associated with subarachnoid haemorrhage (SAH) prompted interest in repair of aneurysms that are discovered before they have ruptured. Several reports prior to 1998 supported this position. Following publication of the initial results of the International Study of Unruptured Intracranial Aneurysms (ISUIA) in $1998,{ }^{1}$ enthusiasm for such intervention was considerably reduced. In this study aneurysms were classified into those in patients with no previous history of SAH (here referred to as "incidental" but called "group l" in the ISUIA report) and those in patients with such a history (here referred to as "additional" but called "group 2" in the ISUIA report). The risk posed by unruptured aneurysms was reported to be lower than had previously been suspected and also the morbidity and mortality of interventions to repair aneurysms was higher than many previously reported series. We published a risk analysis ${ }^{2}$ based on these 1998 findings.

The time has come to revisit the question because of two developments. First, the high penetration achieved by endovascular coiling in the treatment of aneurysms, and secondly the recent publication of updated results from the ISUIA ${ }^{3}$ which now includes 6544 patient years of prospective follow up of untreated unruptured aneurysms, 1917 surgically operated and 451 coiled unruptured aneurysms.

In this analysis we attempt to interpret the 2003 ISUIA results by comparing the outcomes over many years of hypothetical groups of patients with unruptured aneurysms treated either by repair or observation.

It is common for clinicians to make a mental estimate of life years lost or gained when advising on conditions that carry long term natural history risks. These are compared with treatments that also carry risks. Such analysis is prone to two errors that overestimate the benefits of treatment. First, annual risk is simply multiplied by a number of years to gain an overall risk. This is a good approximation for small risks over short times-1\% over 10 years is estimated as $10 \%$, which is close to the real 9.6\%. Larger risks over longer times are, however, overestimated. A $2 \%$ risk over 30 years is actually $45 \%$, considerably less than $60 \%$. The second error is in comparing an immediate risk equally with a risk spread over many years. A long term risk has less impact. As an example of this point, an individual with a 30 year remaining life expectancy taking a $10 \%$ risk now with no associated benefit will lose, on average, 3 years of life expectancy. If that risk is spread over the 30 years they will lose only 1.5 years because if the risk is realised it will on average be in 15 years time, not now. These problems are overcome by the present analysis.

\section{METHOD}

The age specific annual risk of death was taken for the population as a whole from the 2001 census mortality statistics of the total population of the UK. ${ }^{4}$ The risk posed by an unruptured aneurysm was estimated by correcting the bleed rate for the favourable recovery rate from SAH. When added, the resulting total annual risk was used to calculate the remaining life expectancy of individuals harbouring unruptured aneurysms. The life expectancy of those undergoing surgery was calculated by adjusting the life expectancy of the normal population for operative risks. The result was compared with the untreated case to calculate expected life years saved or lost by operating. Further details of the mathematical method are given in the appendix. The annual rupture rates used are given in table 1 and are derived from observations over a maximum period of six years. ${ }^{3}$

\section{Treatment of confidence}

It should be emphasised that the main problems with our conclusions are not connected with chance but with the potential errors discussed below. In order to give an idea of the expected variation due to chance we have included the life years saved and zero crossing ages with $95 \%$ confidence

Abbreviations: ISUIA, International Study of Unruptured Intracranial Aneurysms; SAH, subarachnoid haemorrhage 
Table 1 Bleed rates (\%) from numerical data in 2003 ISUIA calculated as annual bleed risk rounded to two significant figures

\begin{tabular}{|c|c|c|c|c|c|}
\hline & \multicolumn{2}{|l|}{$<7 \mathrm{~mm}$} & \multirow[b]{2}{*}{$7-12 \mathrm{~mm}$} & \multirow[b]{2}{*}{$13-24 \mathrm{~mm}$} & \multirow[b]{2}{*}{$>24 \mathrm{~mm}$} \\
\hline & Incidental & Additional & & & \\
\hline Cavernous carotid & 0 & 0 & 0 & 0.61 & 1.3 \\
\hline $\mathrm{AC} / \mathrm{MC} / \mathrm{IC}$ & $\begin{array}{l}0 \text { (0.08 if posterior } \\
\text { communicating artery included) }\end{array}$ & 0.30 & 0.53 & 3.1 & 9.7 \\
\hline $\mathrm{PC} / \mathrm{P}$ & 0.51 & 0.67 & 3.1 & 4.0 & 13 \\
\hline
\end{tabular}

$A C$, anterior communicating; IC internal carotid; $M C$, middle cerebral; $P$, posterior circulation; $P C$ posterior communicating.

limits for surgically clipped incidental anterior aneurysms (table 2). These $95 \%$ limits are derived from compound probability distributions. Both the rate of rupture of unruptured aneurysms and the rate of poor outcome following treatment were modelled as Poisson processes. A probability was calculated for each combination of bleed rate and poor outcome rate values over the distributions of these variables. For each combination of values the model was run to calculate the life years gained and zero crossing age. The life years and zero crossing ages thus calculated were ranked and cumulative probability distributions calculated from which 95\% limits were obtained. Strictly we should have considered the age specific mortalities of the background population to be randomly distributed variables also but this would have made the calculations intractable, and sensitivity analysis showed that treating them as point estimates led to a less than $1 \%$ error in the $95 \%$ confidence limits.

\section{Data extraction}

Two aneurysm classification schemes were made by the ISUIA in the light of the results: the aneurysm size ranges and anatomical locations. Many previous studies, including the 1998 ISUIA report, classified size ranges as under $10 \mathrm{~mm}$ with two or more larger ranges. This was changed in the 2003 report to under $7 \mathrm{~mm}$ because several bleeds were observed in the 7-9 mm incidental aneurysms. The second scheme was an unconventional anatomical classification of aneurysms into (a) anterior including anterior communicating, middle cerebral, and internal carotid arteries aneurysms and $(b)$ posterior including the posterior circulation and posterior communicating artery aneurysms. In the conventional classification scheme posterior communicating artery aneurysms are grouped with the anterior circulation as they arise from the junction of the internal carotid and the posterior communicating artery. The reason that the unconventional scheme was used was that the haemorrhages observed in small incidental anterior circulation aneurysms (using the term in its conventional sense) were from posterior communicating artery aneurysms. The hypothesis that they are more dangerous than other anterior circulation aneurysms is thus reasonable but as the number of bleeds involved was small

Table 2 Life years saved at age 20 and zero crossing ages for surgical clipping of incidental anterior aneurysms (with 95\% confidence limits)

\begin{tabular}{lll}
\hline Aneurysm size & Life years saved at age 20 & Age of zero crossing \\
\hline$<7 \mathrm{~mm}$ & $-1.3(-1.9$ to 7.7$)$ & $-(-$ to 54$)$ \\
$7-12 \mathrm{~mm}$ & $5.6(-0.5$ to 12.4$)$ & $54(-$ to 62$)$ \\
$13-24 \mathrm{~mm}$ & $25.7(10.6$ to 40$)$ & $64(49$ to 73$)$ \\
$>24 \mathrm{~mm}$ & $38.8(24$ to 43$)$ & $68(53$ to 74$)$ \\
\hline
\end{tabular}

(we estimate two), further corroborative evidence is needed before this hypothesis can be accepted with confidence.

Further details of the ISUIA results, particularly on natural history, are expected soon.

\section{Risk of rupture}

Data in the 2003 ISUIA report are given as the risk of SAH over various time periods for various subgroups of aneurysms. From these we have calculated the annual risks shown in tables 1 and 3. Annual risks from risks over longer periods were calculated as $a=\left(1-(1-r)^{1 / t}\right)$ where $a$ is the annual risk and $r$ is the given risk over time period $t$ in years. Rounding is to two significant figures. Cumulative risks for different subgroups are given as numbers in the ISUIA report from which the rupture rates given in table 1 are derived and in graphs from which the rates given in table 3 are derived. Because the risks of posterior communicating artery aneurysms are open to debate we have highlighted the distinction between grouping them with the anterior and posterior circulations.

\section{Operative risk}

In the interventional part of the ISUIA, morbidity and mortality for clipping and coiling unruptured aneurysms were calculated from 1917 surgical and 451 endovascular cases. Results were stratified according to patient age, aneurysm size, and anatomical location. The categories according to size were $<13 \mathrm{~mm}, 13-24 \mathrm{~mm}$ and $>24 \mathrm{~mm}$. The $<13 \mathrm{~mm}$ group was not split into $<7 \mathrm{~mm}$ and $7-12 \mathrm{~mm}$ as was the case for the natural history part of the study. This was because there was no significant difference between them. Weighted means were used to ascribe an operative risk to each aneurysm subgroup so that the risk used was specific to size and location.

The division of outcome into convenient "good" and "bad" groups is not straightforward because the ratio of the morbidity to mortality is different for the haemorrhagic group and the operative group. About $86 \%$ of those with a

Table 3 Bleed rates for all locations calculated as annual bleed risk rounded to two significant figures

\begin{tabular}{lc}
\hline $\begin{array}{l}\text { Groups by } \\
\text { aneurysm size }\end{array}$ & $\begin{array}{l}\text { Annual risk } \\
\text { of rupture (\%) }\end{array}$ \\
\hline Annual rates of rupture for incidental aneurysms (ISUIA) \\
$<7 \mathrm{~mm}$ & 0.10 \\
$7-12 \mathrm{~mm}$ & 1.5 \\
$13-24 \mathrm{~mm}$ & 2.7 \\
$>24 \mathrm{~mm}$ & 5.3 \\
Annual rates of rupture for additional aneurysms (ISUIA) \\
$<7 \mathrm{~mm}$ & 0.4 \\
$7-12 \mathrm{~mm}$ & 0.8 \\
$13-24 \mathrm{~mm}$ & 1.2 \\
\end{tabular}



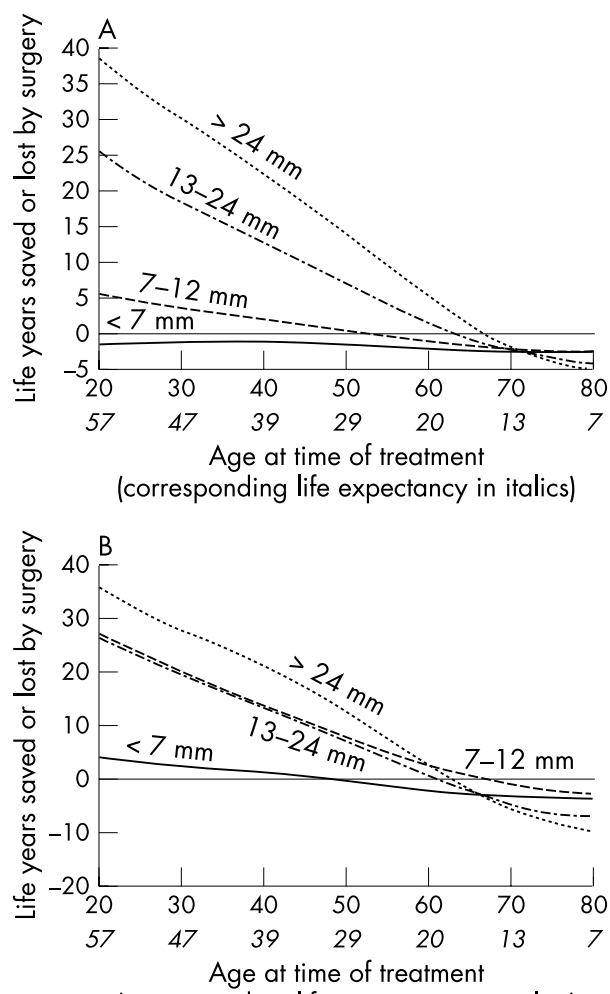

(corresponding life expectancy in italics)

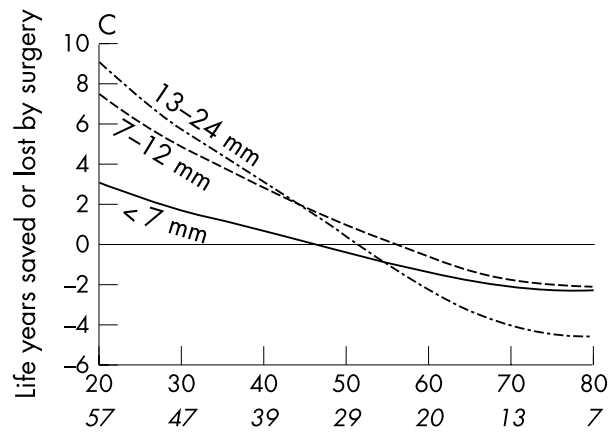

Age at time of treatment

(corresponding life expectancy in italics)

Figure 1 Expected life years lost or gained by treatment against patient age at the time of treatment for surgical treatment of unruptured aneurysms in four size ranges: (A) Incidental aneurysms (no previous history of subarachnoid haemorrhage (SAH)) of the anterior circulation (internal carotid, anterior cerebral, middle cerebral (including posterior communicating) arteries). Note that the bleed rate used for the anterior circulation $<7 \mathrm{~mm}$ group is not $0 \%$ per year as given by ISUIA but $0.08 \%$ calculated by including the posterior communicating aneurysms. (B) Incidental aneurysms of the posterior circulation (excluding posterior communicating artery) and (C) additional (previous $\mathrm{SAH}$ ) aneurysms. Anatomical groups are not separated.

bad outcome following an SAH will be dead whereas only about $25 \%$ of those with a bad outcome following operative treatment will be dead. This complicates the task of selecting comparable groups. The compromise we used was to count deaths and those requiring care every day or more frequently (modified Rankin 3-5) one year after the operation as bad outcomes and the rest as good. Size and location specific rates of morbidity and mortality are taken from the ISUIA data. ${ }^{3}$

The annual risk posed by an aneurysm was estimated by multiplying the bleed rates given in the ISUIA report by the "bad outcome rate" following SAH. Reported series and previous risk analyses have divided outcomes following SAH into death or serious disability and full recovery or mild
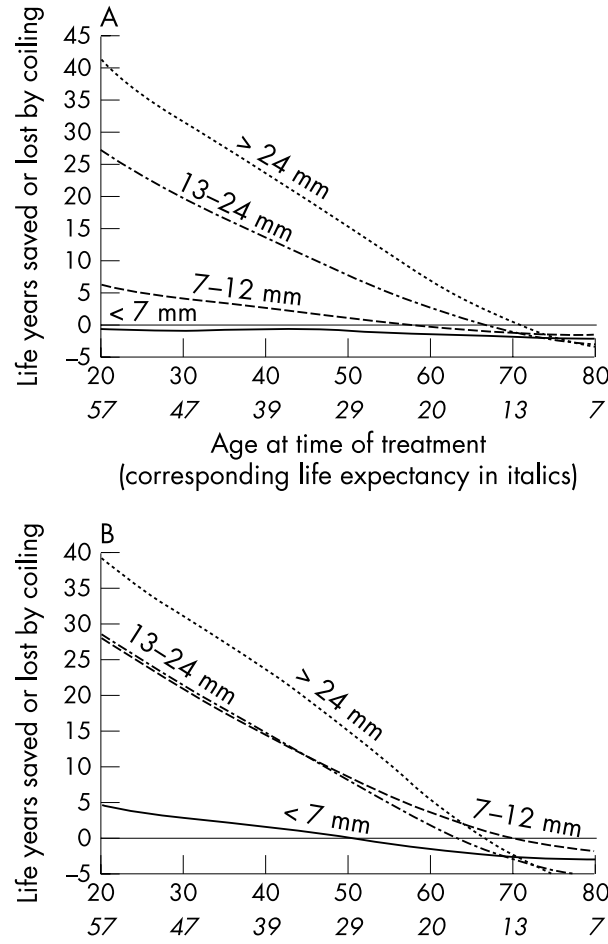

Age at time of treatment

(corresponding life expectancy in italics)

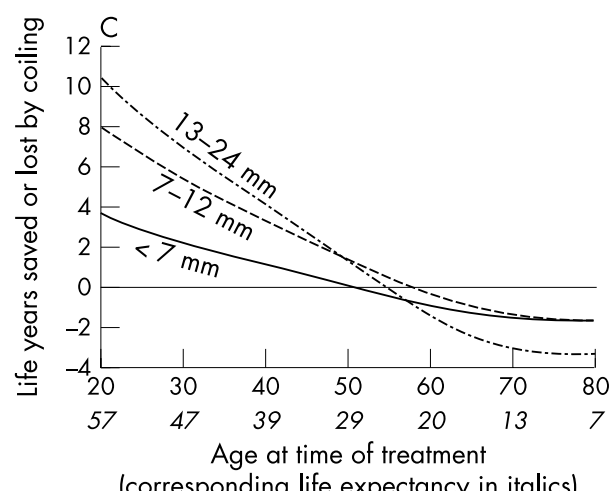

(corresponding life expectancy in italics)

Figure 2 Expected life years lost or gained by treatment against patient age at the time of treatment for endovascular embolisation of unruptured aneurysms in four size ranges. (A) Incidental aneurysms (no previous history of subarachnoid haemorrhage (SAH)) of the anterior circulation (internal carotid, anterior cerebral, middle cerebral (including posterior communicating) arteries). (B) Incidental aneurysms of the posterior circulation and (C) additional aneurysms. Implicit in the results presented in these graphs is the assumption that embolisation provides $100 \%$ long term protection from aneurysm rupture, an assumption that may prove to be false.

disability and the convention is followed. Serious disability is usually classified as requiring assistance with the activities of daily living. A modified Rankin score of 3-5 was used by ISUIA and is also used here. Death was the result of $65 \%$ of haemorrhages in the 2003 ISUIA report. ${ }^{3}$ Morbidity was not given but other studies of outcome following SAH suggest that $30 \%$ of survivors have "poor" neurological recovery..$^{5-8}$ Combining these figures leads to an overall bad outcome rate following rupture of an aneurysm under follow up of $75 \%$. The bleed risk is scaled by this factor before adding it to the population's age specific mortality to obtain the overall risk. The bleed risk was assumed to remain constant over time. 


\section{RESULTS}

There are 22 combinations of the three variables: aneurysm size range, clipping or coiling, and incidental or additional. This leads to 22 plots of expected life years lost or gained by treatment against patient age or remaining life expectancy (figs 1 and 2). These plots have many common features. The number of life years gained by repairing aneurysms declines with increasing age of the patient at the time of repair. For all types of aneurysm there is an age beyond which repair results in a loss of expected remaining life years. The lowest rate of rupture is seen for incidental anterior circulation aneurysms under $7 \mathrm{~mm}$ in diameter. Both surgical and endovascular treatment of these aneurysms results in a net loss of life years at all ages over 20 . For the other 20 combinations of aneurysm and treatment type, life years are gained by operating where life expectancy is greater than 15-45 years. For the normal population this corresponds to ages under 45 and 70 years. In general, aneurysms with a more dangerous natural history (large ones; posterior circulation aneurysms) are also more dangerous to treat but the natural history risks dominate and treating more dangerous aneurysms leads to greater benefits. Any aneurysm over $24 \mathrm{~mm}$ in diameter shows around a 40 year improvement in life expectancy from treatment at age 20 and some improvement from treatment up the around 70 years of age. On the other hand an aneurysm under $7 \mathrm{~mm}$ in diameter shows more modest gains of under five years from treatment at age 20 with benefit up to around age 50, with the exception of incidental anterior aneurysms which show no benefits. It is worthy of note that while the number of life years saved at young ages is highly variable the age at which benefit becomes loss (the age of zero crossing) is less so. This is because the age of zero crossing is largely determined by the rate at which operative risks rise with age.

\section{DISCUSSION}

The present calculations are based on three statistics: the life expectancy of the normal population, the risks posed by untreated unruptured aneurysms, and the poor outcome rate of procedures to repair unruptured aneurysms. None of these values are known exactly.

We first consider the life expectancy of the normal population, which is quite accurately known now, and we do not believe that errors from this source are significant. There are, however, two provisos. First, current figures are largely based on the death rates of older people. We do not know whether today's figures will truly reflect the life expectancy of those now in their thirties and forties, the age range to which our calculations are largely geared. Secondly, the assumption that persons with aneurysms have equal life expectancy to those without if the aneurysms are secured may not be correct. These problems can be circumvented by considering remaining life expectancy rather than age but this brings in another assumption that could be contested. The calculations use age specific surgical risks and the rise in risk with age largely determines the age of zero crossing. If the remaining life expectancy of a particular 40 year old is the same as that of the normal population at 60 , is their surgical risk that of a 40 year old or a 60 year old? The assumption is that it is that of a 60 year old.

The other two statistics we used are based primarily on data from the ISUIA and thus stand or fall on their accuracy. ISUIA has measured the bleed risk for different size ranges (table 1) and for different anatomical locations of aneurysms. The very low bleed rate from small incidental aneurysms is important because many presenting unruptured aneurysms fall into this group and it implies that no treatment should be undertaken. Earlier reports suggested a 1-2\% annual bleed risk though the discrepancy is not as large as it appears. We have pooled studies published before 1998. These studies contained about 3500 patient years of follow up of unruptured aneurysms, over $65 \%$ of which were additional aneurysms. Retrospective examination of these data reveals around 835 life years of follow up in patients with incidental aneurysms under $10 \mathrm{~mm}$ in diameter and in this subgroup the risk is indeed small at $0.24 \%$ haemorrhage per patient year. This figure is still larger than the 2003 ISUIA figure of $0.1 \%$ but both figures are based on one or two haemorrhages over the observation period. Wide variations due to chance are thus to be expected.

However, there are other concerns. The safe size range reported in the 1998 results was under $10 \mathrm{~mm}$. This has been revised post hoc to under $7 \mathrm{~mm}$ because five ruptures were noted in the $7-9 \mathrm{~mm}$ size range. The denominator of observed patient years in this 7-9 $\mathrm{mm}$ group is not given. The classification of site is also unusual and was decided on in the light of the results. Both of these changes appear to have the effect of reinforcing the theory that there exists a group of small incidental aneurysms that are safe. With the present evidence this theory does seem to be correct but does not marry well with the other ISUIA data which can be seen from table 3 to give lower bleed rates in all other size ranges to additional aneurysms. Even with these provisos it appears that the under $7 \mathrm{~mm}$ incidental aneurysms of the anterior circulation do present a very low bleed risk but should posterior communicating artery aneurysms be included or not?

Several reports show aneurysms of the posterior circulation to carry a higher haemorrhage risk than those of the anterior circulation. ${ }^{19}{ }^{10}$ Consistent differences in risk between aneurysms at the three main anterior sites (anterior cerebral, internal carotid including posterior communicating, and middle cerebral) have not previously been reported but ISUIA classified posterior communicating artery aneurysms in the posterior rather than anterior group. ${ }^{3}$ This was because the bleeds from small incidental anterior aneurysms (we estimate there were two) were both from posterior communicating aneurysms. Because the hypothesis that posterior communicating aneurysms behave like posterior circulation aneurysms was proposed post hoc it requires corroboration on a different data set before it can be accepted. Most reports on the natural history of unruptured aneurysms do not

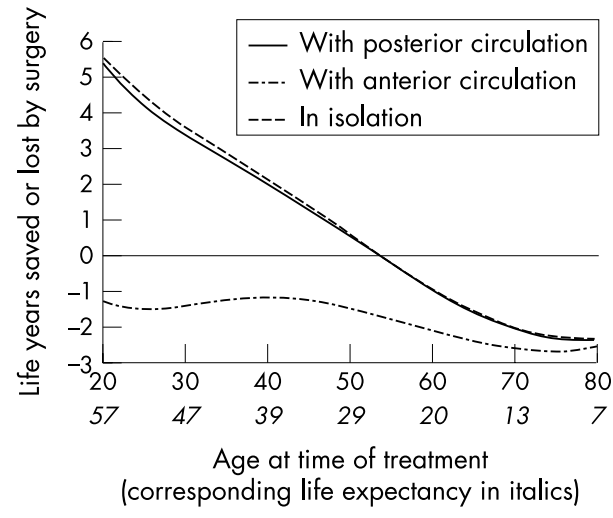

Figure 3 What do we do with posterior communicating artery aneurysms? This figure shows the effect of including posterior communicating artery aneurysms with the anterior circulation as per convention (giving a bleed rate of $0.08 \%$ per year for incidental aneurysms $<7 \mathrm{~mm}$ ) or with the posterior circulation as per the 2003 ISUIA report (giving a bleed rate of $0.51 \%$ per year for incidental aneurysms $<7 \mathrm{~mm}$ ), or on their own (giving a bleed rate of $0.53 \%$ per year for aneurysms $<7 \mathrm{~mm}$ ). The similarity between the posterior communicating and posterior bleed rates seems to suggest they belong together but there is insufficient evidence to accept the hypothesis. 
provide a breakdown into anatomical locations. In one study that does, further breakdown into size and incidental/ additional is not given. ${ }^{9}$ Here the bleed rate of posterior communicating aneurysms was very similar to other anterior locations and around 10 times lower than posterior locations. The hypothesis thus fails.

An alternative to grouping posterior communicating aneurysms with the anterior or posterior circulations would be to treat them in isolation but this has the same problemthat the hypothesis that they are different from other anterior aneurysms is also a post hoc product of the ISUIA data set. There is a substantial probability $(p=0.23)$ that two bleeds occur in the same anterior circulation subgroup by chance alone. Their observation cannot therefore be taken as good evidence that the subgroup (posterior communicating in this case) is in any way special. We consider that the case for grouping posterior communicating aneurysms with the posterior circulation or for treating them in isolation is weak and prefer to default to the "null hypothesis" of anterior aneurysms belonging together on anatomical and historical grounds. This gives an overall risk of $0.08 \%$ per year for incidental anterior circulation aneurysms $<7 \mathrm{~mm}$ rather than the $0 \%$ reported for the anterior cerebral/internal carotid/middle cerebral group (fig 3).

It should be emphasised that the conclusion that small anterior incidental aneurysms should not be treated is largely dependent on this low rate being accepted. It would be difficult to displace the conclusion by arguing from surgical results better than those of ISUIA. In order to show no benefit or loss at 20 years of age in these small incidental aneurysms, the unfavourable outcome rate from treatment would have to be $30 \%$ lower than those of ISUIA and to gain one life year at age 20 it would have to be $75 \%$ lower.

Data on outcomes following surgical or endovascular treatment remain controversial. Several series have been reported with substantially lower morbidity and mortality than found by the ISUIA. Greater note was taken of cognitive impairment in the ISUIA than had been done previously and this accounts to some extent for the results being poorer than in other earlier series, ${ }^{11-14}$ but there remain more recent reports with similar outcome criteria and better results ${ }^{15} 16$ though the numbers of cases are small. This has led to the suggestion that specialist neurovascular centres can perform significantly better than the ISUIA result would indicate. Against this must be weighed the apparent quality of this part of the ISUIA data. Outcomes were collected from multiple centres, the numbers involved were large and the operative outcome data were collected prospectively with independent assessment. While it may be appropriate for certain well performing specialist centres to make judgements on treatment based on their own results rather than those of the ISUIA, we feel such a course should not be recommended unless such results are independently assessed and are statistically significantly different from those of ISUIA.

There are two critical assumptions that, if proved incorrect, would lead to a significant change in the calculated age of no benefit. The first is that the annual bleed risk posed by an aneurysm remains constant for the remainder of a patient's life. The 2003 ISUIA report implies that this is not true. Of the 51 haemorrhages that were observed, 49 occurred within five years of diagnosis of the aneurysm. The implication is that aneurysms bleed risks fall with time from diagnosis. However, the information to confirm this claim is not given in the report. It is crucial to know how many patient years of follow up there were in the pre and post five year time bands and this is not given. We have made estimates by interpolation from values given graphically and these estimates of the pre and post 5 year bleed rates are not significantly different.
Other series reported with more complete data, notably that of Heiskenan and Juvela et al, ${ }^{17}{ }^{18}$ suggesting that the rate of aneurysm rupture does remain relatively constant over time. While the numbers involved in Heiskenan's series are not as large as in the ISUIA, it is a substantial study with 2575 patient years of follow up which extends in some cases to over 40 years.

The other critical assumption which, if false, would substantially change our results is that the observed SAH in the conservatively treated ISUIA group did indeed originate from the aneurysms detected earlier. If a significant proportion of them originated rather from de novo aneurysms, this would clearly reduce the benefit of surgical or endovascular treatment.

The endovascular treatment calculations are susceptible to a further assumption being refuted in the future. This is that coiling provides permanent protection from aneurysm rupture. Data on this are currently being collected but are not yet available. The same reservation could be applied to surgical clipping but it has been in common use for more than 40 years and post-clipping bleeds are confined to a handful of case reports.

One factor concerning bleed risk that has still to be fully considered is the efficacy of medical treatment. Slosberg found a low rate of rupture from previously ruptured aneurysms treated with careful hypotensive regimens. ${ }^{19} 20$ Many of his patients harboured aneurysms with a high risk of haemorrhage. They were treated conservatively because they were considered to pose unacceptably high surgical risk. Thus it remains unknown whether medical treatment for unruptured aneurysms is superior or inferior to endovascular or surgical treatment.

\section{CONCLUSIONS}

Based on the results of the ISUIA, the impact on life expectancy of repairing unruptured intracranial aneurysms varies from a substantial benefit to a modest detriment. In patients with incidental anterior circulation aneurysms under $7 \mathrm{~mm}$ in diameter repairing the aneurysm brings a slight reduction in life expectancy at all ages. In all patients with additional aneurysm and those with incidental aneurysms $7 \mathrm{~mm}$ or more in diameter or of the posterior circulation, life years are gained by repair up to the age of 45-65. These results are dependent on the accuracy of the ISUIA data.

\section{Authors' affiliations \\ R R Vindlacheruvu, A D Mendelow, P Mitchell, Department of Neurosurgery, Newcastle General Hospital, Newcastle upon Tyne, UK Competing interests: none declared}

\section{APPENDIX}

\section{MATHEMATICAL METHOD}

The annual risk posed by an unruptured aneurysm $(r)$ was taken as the bleed rate multiplied by the bad outcome rate for $\mathrm{SAH}$ as discussed above. The age specific mortality $(m)$ of the background population had to be approximated by a continuous function because the statistics were only available for five year age ranges rather than the one year resolution needed. It was further necessary that the gradient of the approximating function was strictly positive over the age range in question. This was approximated by an exponential function of a $4^{\circ}$ polynomial giving a residual variance of 0.002 deaths $2 / 1000$ person years. Polynomial and exponential polynomial functions of $1-6^{\circ}$ were tried and this gave the lowest residual variance without having any negative gradient; $r$ was added to this function giving a total annual risk $(M)$ for those harbouring unruptured aneurysms 
$(M=m+r)$. Survival to age $x$ (which will be called $S(x))$ after diagnosis at age $a$, is then calculated using the recurrence relation: $S(x)=(1-M(x)) S(x-1), S(a)$ being 1 . Remaining life expectancy at age $a$ is then given by:

$$
\int_{a}^{\infty} S(x) d x
$$

Life expectancy of a patient with an aneurysm that has been repaired successfully is assumed to be normal. The operative morbidity and mortality are included by assuming an initial survival of $\left(1-r_{0}\right)$ where $r_{0}$ is the operative risk of a bad outcome. $r_{0}$ is an age dependent interpolation of ISUIA surgical and endovascular outcome data. All calculations were done numerically with the program "Matlab 6.5" running on a PC. The quadrature method of integration was used.

\section{REFERENCES}

1 Anonymous. Unruptured intracranial aneurysms - risk of rupture and risks of surgical intervention. International Study of Unruptured Intracranial Aneurysms Investigators. N Engl J Med 1998;339:1725-33.

2 Mitchell P, Jakubowski J. Risk analysis of treatment of unruptured aneurysms. J Neurol Neurosurg Psychiatry 2000;68:577-80.

3 Anonymous. Unruptured intracranial aneurysms: natural history, clinical outcome, and risks of surgery and endovascular treatment. Lancet 2003;362:103-10.

4 Census 2001. National Statistics Office (UK) 2001.

5 Deruty R, Patet JD, Mottolese C, et al. Long-term outcome of the management of ruptured intracranial aneurysm. Review of 328 consecutive patients treated over a period of 12 years. Neurol Res 1988;10:217-20.
6 Liunggren B, Fodstad H, von Essen C, et al. Aneurysmal subarachnoid haemorrhage: overall outcome and incidence of early recurrent haemorrhage despite a policy of acute stage operation. Br J Neurosurg 1988;2:49-53.

7 Saveland H, Hillman J, Brandt L, et al. Overall outcome in aneurysmal subarachnoid hemorrhage. A prospective study from neurosurgical units in Sweden during a 1-year period. J Neurosurg 1992;76:729-34.

8 Saveland $\mathrm{H}$, Brandt $\mathrm{L}$. Which are the major determinants for outcome in aneurysmal subarachnoid hemorrhage? A prospective total management study from a strictly unselected series. Acta Neurol Scand 1994;90:245-50.

9 Wiebers DO, Whisnant JP, Sundt TM Jr, et al. The significance of unruptured intracranial saccular aneurysms. J Neurosurg 1987;66:23-9.

10 Rinkel GJ, Diibuti M, Algra A, et al. Prevalence and risk of rupture of intracranial aneurysms: a systematic review. Stroke 1998;29:251-6.

11 Mizoi K, Suzuki J, Yoshimoto T. Surgical treatment of multiple aneurysms. Review of experience with 372 cases. Acta Neurochir (Wien) 1989:96:8-14.

12 Mizoi K, Yoshimoto T, Nagamine Y, et al. How to treat incidental cerebral aneurysms: a review of 139 consecutive cases. Surg Neurol 1995;44: 114-20; discussion 120-1.

13 Solomon RA, Fink ME, Pile-Spellman J. Surgical management of unruptured intracranial aneurysms. J Neurosurg 1994;80:440-6.

14 Yoshimoto T, Mizoi K. Importance of management of unruptured cerebral aneurysms. Surg Neurol 1997;47:522-5; discussion 525-6.

15 Ohue S, Oka Y, Kumon Y, et al. Importance of neuropsychological evaluation after surgery in patients with unruptured cerebral aneurysms. Surg Neurol 2003;59:269-75; discussion 275-6.

16 Hempelmann RG, Barth $H$, Buhl R, et al. Clinical outcome after surgery of intracranial unruptured aneurysms: results of a series between 1991 and 2001. Acta Neurochir Suppl 2002;82:51-4.

17 Juvela S, Porras M, Poussa K. Natural history of unruptured intracranial aneurysms: probability of and risk factors for aneurysm rupture. J Neurosurg 2000;93:379-87

18 Heiskanen 0 . Risk of bleeding from unruptured aneurysm in cases with multiple intracranial aneurysms. J Neurosurg 1981;55:524-6.

19 Slosberg PS. Unexpected results in long-term medically treated ruptured intracranial aneurysm including data on 14 patients followed more than 30 years each. Acta Neurochir (Wien) 1997; 139:697-705.

20 Slosberg PS. Zero percent mortality due to recurrent hemorrhage in follow-up of medically treated ruptured single intracranial aneurysms: a 23-year study. Trans Am Neurol Assoc 1979;104:180-3. 